\title{
FORMAS DE ARMAZENAMENTO DE SEMENTES DE ARAÇAZEIRO
}

Alexandre Hack Porto, Américo Wagner Júnior, Carlos Kosera Neto, Marciéli da Silva, Alberto Ricardo Stefeni, Gisely Correa de Moura

Universidade Tecnológica Federal do Paraná - UTFPR, Programa de Pós-Graduação em Agronomia, câmpus Pato Branco e Dois Vizinhos, PR. E-mail: alexandrehack@gmail.com

\section{RESUMO}

$\mathrm{Na}$ flora arbórea brasileira destaca-se o araçazeiro, fruteira nativa da família Myrtaceae com grande potencial de produção. Na propagação predomina o uso de sementes, pois apresenta grande homogeneidade no padrão das mudas obtidas, relacionado a apomixia e curto período juvenil. Entretanto, é necessário adotar técnicas de armazenamento que garantam a viabilidade por maior período de tempo. Neste sentido, o trabalhou objetivou avaliar formas de conservação da viabilidade de sementes de araçazeiro amarelo cv. Ya-Cy por período de 12 meses. Adotou-se delineamento experimental inteiramente casualizado (DIC), em bifatorial $12 \times 5$ (tempo x formas de armazenamento), com quatro repetições de 100 sementes. Foram avaliadas a porcentagem de germinação ( $G$ \%), índice de velocidade de germinação (IVG) e tempo médio de germinação (TMG). Recomendou-se o uso de recipientes como eppendorf ${ }^{\circledR}$ ou material tetrapak ${ }^{\circledast}$ para armazenar sementes de araçazeiro $\mathrm{Ya}-\mathrm{Cy}$, visando conservar sua viabilidade e vigor até os 330 dias. Utilização de gordura animal não foi recomendada para armazenamento de sementes de araçazeiro $\mathrm{Ya}-\mathrm{Cy}$.

Palavras-chave: Araça amarelo; Psidium cattleyanum Sabine; viabilidade de sementes; conservação de sementes.

\section{FORMS OF STORAGE OF ARAÇAZEIRO SEEDS}

\begin{abstract}
In the Brazilian tree flora stands the araça tree, native fruit of the family Myrtaceae with great potential of production. In the propagation, the use of seeds predominates, since it presents great homogeneity in the pattern of the seedlings obtained, related to apomixis and short juvenile period. However, it is necessary to adopt storage techniques that guarantee the viability for a longer period of time. In this sense, the objective of this work was to evaluate the viability conservation of yellow arachnid cv. Ya-Cy for a period of 12 months. A completely randomized experimental design (DIC) was used in a $12 \times 5$ two-phase experiment (time $x$ storage forms), with four replicates of 100 seeds. The percentage of germination (G\%), germination speed index (IVG) and average germination time (TMG) were evaluated. It is recommended to use containers such as eppendorf ${ }^{\circledast}$ or tetrapak ${ }^{\circledR}$ material to store Ya-Cy arachnid seeds, in order to preserve their viability and vigor until 330 days. Use of animal fat is not recommended for storage of Ya-Cy araçazeiro seeds.
\end{abstract}

Keywords: Yellow Araça; Psidium cattleyanum Sabine; viability seed; conservation seed.

\section{INTRODUÇÃO}

A flora arbórea presente no Brasil é a mais diversificada do mundo, com presença de várias espécies de fruteiras nativas com potencial para uso na agricultura, já que apresentam frutos comestíveis muito apreciados pela população nas regiões que ocorrem (KOHAMA et al., 2006; DANNER, 2009; DANNER et al., 2010). Dentro desta grande diversidade destaca-se $\mathrm{o}$ araçazeiro (Psidium cattleyanum Sabine), pertencente à família Myrtaceae (TOMAZ et al., 2011), com ampla área de ocorrência situada na Costa Atlântica, que se estende desde o norte Brasileiro até a região Sul do Rio Grande do Sul, chegando ao Nordeste do Uruguai (CASTRO et al., 2004). 
O potencial desta fruteira é imenso e está intrinsicamente ligado a suas características fitoquímicas, cujas propriedades as tornam interessantes para indústria (MARIN et al., 2014), como a presença de compostos antioxidantes (NERI-NUMA et al., 2013). O suco de sua fruta apresenta elevado teor de compostos fenólicos, com preservação do teor vitamina $\mathrm{C}$ em torno de $45 \%$ (FRANZON et al., 2009). Além disso, pode ser utilizado para o consumo in natura ou na fabricação de derivados a exemplo de licores, fermentados, geleias, doces, sorvetes, picolés, condimentos (VIEIRA et al., 2006; BRACK et al., 2007). A incorporação do araçá junto a cadeia produtiva de frutas pode trazer diversificação para o mercado consumidor, com a inclusão de espécies nativas incentivando a exploração sustentável e a subsistência de comunidades tradicionais em diversas regiões do país (FRANZON et al., 2009). Para incentivar o cultivo desta espécie duas cultivares foram lançadas pela Embrapa Clima Temperado, uma de película amarela, "Ya-cy", e outra vermelha, "Irapuã", embora em pequena escala, estas cultivares vem sendo cultivadas em pomares comerciais (FRANZON, 2004). A cultivar $Y a-C y$ apresenta destaque por propiciar duas a três colheitas por ano e ter frutos de boa aceitabilidade pelo mercado consumidor (ZANELA et al., 2012). Isso possibilita o aumento na demanda por mudas de qualidade.

A propagação do araçazeiro é predominantemente seminífera (MANICA, 2000), dada à importância da propagação sexuada para esta espécie por apresentar grande homogeneidade no padrão das mudas obtidas, o que pode ser relacionado a apomixia e curto período juvenil, ou seja, mesmo por sementes as plantas produzem no segundo e terceiro ano. Todavia, dependendo da forma de armazenamento ou do ambiente para semeadura, a semente pode ter seu processo germinativo comprometido, tornando-se necessário a adoção de técnicas que garantam manter sua viabilidade por maior período de tempo (WIELEWICKI et al., 2006).

Para que o produtor de mudas tenha disponibilidade de material por maior período, mantendo regularidade na produção das mudas, as técnicas de armazenamento devem ser aprimoradas, para que haja eficácia no processo. Neste sentido, com o presente trabalho objetivou avaliar formas de conservação da viabilidade de sementes de araçazeiro amarelo cv. $\mathrm{Ya}$-Cy por período de 12 meses.

\section{MATERIAL E MÉTODOS}

O experimento foi conduzido no Laboratório de Fisiologia Vegetal, da Universidade Tecnológica Federal do Paraná Câmpus Dois Vizinhos. Foram coletados frutos de Psidium catlleyanum Sabine, do Pomar de Fruteiras Nativas da referida instituição. Os frutos apresentavam-se em estádio de maturação fisiológica, com as características de coloração totalmente amarela da epiderme.

Os frutos foram despolpados e as sementes extraídas manualmente usando água corrente em peneira de aço com malha fina, realizando leve friç̧ão até o desprendimento total da polpa, com posterior secagem sobre papel toalha a sombra em temperatura ambiente por 48 horas.

Para a caracterização do lote foi realizado o peso de mil sementes (PMS), calculado segundo a RAS (BRASIL, 2009), onde obteve a massa de 45.234 gramas.

Foi adotado delineamento experimental inteiramente casualizado (DIC), em bifatorial $12 \mathrm{X}$ 5 (tempo $x$ formas de armazenamento), com quatro repetições de 100 sementes. Como tempo de armazenamento foram realizadas avaliações ao zero, 30, 60, 90, 120, 150, 180, 210, 240, 270, 300 e 330 dias. No tempo zero, as sementes foram submetidas ao teste de germinação logo após sua secagem a sombra, consistindo no tratamento controle.

As formas de armazenamento utilizadas propiciaram testar condições de atmosfera modificada através do saco plástico com dimensões de $15 \times 15 \times 80 \quad(\mathrm{~cm} \quad x \quad \mathrm{~cm} \quad x \quad \mu)$ submetidos a vácuo; copo plástico de $50 \mathrm{~mL}$ onde as sementes foram acondicionadas e recobertas com gordura animal; envelope moldado a partir de caixa de leite tipo Tetrapak ${ }^{\circ}$ de dimensões 10 $x 10 \mathrm{~cm}$; tubos tipo Eppendorf ${ }^{\circ}$ de $5 \mathrm{~mL}$; sacos de papel acondicionadas dentro de lata de alumínio (1 L). As embalagens foram mantidas em condições de temperatura ambiente alocadas em prateleiras de laboratório ao abrigo da luz direta e umidade.

Após cada período de armazenamento, as sementes de cada condição de conservação foram submetidas ao teste de germinação sobre papel Germitest ${ }^{\circ}$, em caixas de poliestireno transparente $(11 \times 11 \times 3 \mathrm{~cm})$ com tampa e mantidas, alocadas em B.O.D. (oxygen 
biochemical demand) sem fotoperíodo, com temperatura controlada $(25 \pm 2 \circ \mathrm{C})$, por 60 dias a partir da semeadura, procedendo-se contagens diárias das sementes que apresentavam protrusão da radícula.

Foram avaliadas a porcentagem de germinação (G \%), índice de velocidade de germinação (IVG) (MAGUIRE, 1962), e tempo médio de germinação (TMG). Com as seguintes fórmulas: $\mathrm{G} \%=\left(\mathrm{n}_{\mathrm{g}}{ }^{*} 100\right) / \mathrm{n}_{\mathrm{s}} ; \mathrm{IVG}=\Sigma\left(\mathrm{n}_{\mathrm{i}} / \mathrm{t}_{\mathrm{i}}\right) ; \mathrm{TMG}=$ $\left(\Sigma n_{i} / t_{i}\right) / \Sigma n_{i}$ em que: " $n_{g}$ " é o número de sementes germinadas ao final do teste; " $n_{s}$ " é o número total de sementes da unidade experimental; " $n_{i}$ " é o número de sementes que germinaram no tempo " "; " $\mathrm{t}_{\mathrm{i}}$ " é o tempo após instalação do teste; " $i "=1 \rightarrow 60$.

Os dados foram submetidos ao teste de normalidade de Shapiro-Wilk, após utilizou-se a transformação Box e Cox (1964) ajustando a equação com o valor de lambda $(\lambda)$ de 0,3 , para as três variáveis analisadas. Em seguida, foram submetidas a análise de variância e comparação de médias pelo teste de Duncan $(\alpha=0,05)$, para fator qualitativo e regressão para o quantitativo, utilizando o programa computacional R (R CORE TEAM, 2017) versão 3.4.1, com auxílio do pacote estatístico ExpDes (FERREIRA et al., 2013).

\section{RESULTADOS E DISCUSSÃO}

Houveram interações significativas entre tempo $\mathrm{X}$ forma de armazenamento para todas as variáveis analisadas. Aos 0 dias de armazenamento obteve-se pouco mais que $50 \%$ de germinação (Tabela 1), resultado que manteve-se estável quando submetidos ao armazenamento durante todo o experimento (330 dias), na maioria das formas de armazenamento, exceto para o tratamento com gordura animal que inviabilizou as sementes de $P$. cattleyanum após 30 dias de armazenamento.

Tabela 1. Porcentagem de germinação de sementes de Psidium catlleyanum armazenados em embalagem a vácuo, gordura animal, envelope Tetrapak ${ }^{\bullet}$, eppendorf e lata de alumínio por 330 dias com avaliações mensais.

\begin{tabular}{|c|c|c|c|c|c|}
\hline $\begin{array}{c}\text { Tempo } \\
\text { (dias) }\end{array}$ & Emb. Vácuo & Gordura & Env. Tetrapak $^{\circledR}$ & Eppendorf & Lata alumínio \\
\hline 0 & 51,34 ab A* & 51,34 a $A$ & 51,34 a $A$ & 51,34 a $A$ & 51,34 ab A \\
\hline 30 & 69,46 a $A$ & $2,97 \mathrm{~b} \mathrm{~B}$ & 57,88 a $A$ & 73,48 a $A$ & 73,32 a $A$ \\
\hline 60 & 71,48 a $A$ & $0,0 \mathrm{~b} \mathrm{~B}$ & 67,09 a $A$ & 65,45 a $A$ & 70,72 a $A$ \\
\hline 90 & 71,37 a $A$ & 0,0 b B & 74,43 a $A$ & 75,09 a $A$ & 77,33 a $A$ \\
\hline 120 & 73,25 a A & 0,0 b B & 75,24 a $A$ & 74,84 a $A$ & 77,22 a $A$ \\
\hline 150 & 36,66 b B & 0,0 b C & 66,99 a $A$ & 64,85 a $A$ & 78,38 a $A$ \\
\hline 180 & 42,88 b B & 0,0 b C & 65,07 a $A$ & 74,37 a $A$ & 69,86 a $A$ \\
\hline 210 & 69,93 a $A$ & 0,0 b B & 52,51 a $A$ & 70,74 a $A$ & 67,32 a $A$ \\
\hline 240 & 74,04 a $A$ & 0,0 b B & 71,69 a $A$ & 71,96 a $A$ & 55,75 ab A \\
\hline 270 & 76,49 а $A$ & 0,0 b B & 67,71 a $A$ & 59,89 a $A$ & 76,39 a A \\
\hline 300 & 50,64 ab A & 0,0 b B & 64,47 a $A$ & 63,57 a $A$ & 65,21 ab A \\
\hline 330 & 67,16 a $A$ & 0,0 b B & 63,72 a $A$ & 55,75 a $A$ & 44,25 b A \\
\hline
\end{tabular}

*Médias seguidas por letras distintas, minúsculas na coluna e maiúsculas na linha, diferem significativamente pelo teste de Duncan $(\alpha=0,05)$.

Porém, quando analisados de maneira isolada, as formas de armazenamento (Figura 1), o vácuo, envelope de Tetrapak e o tubo Eppendorf ${ }^{\circ}$, não apresentaram diferença estatística ao longo do período de armazenamento permanecendo em média de
$65 \%$ de geminação. Entretanto, a gordura perdeu a viabilidade aos 30 dias e quando armazenadas em lata de alumínio atingiu o ponto de máxima eficiência no armazenamento com 148 dias, e obtendo-se assim, 76 \% de germinação. 
Figura 1. Porcentagem de germinação em função do tempo de armazenamento em dias em vácuo (a), gordura (b), env. tetrapak ${ }^{\oplus}$ (c), eppendorf ${ }^{\bullet}(d)$, lata (e).
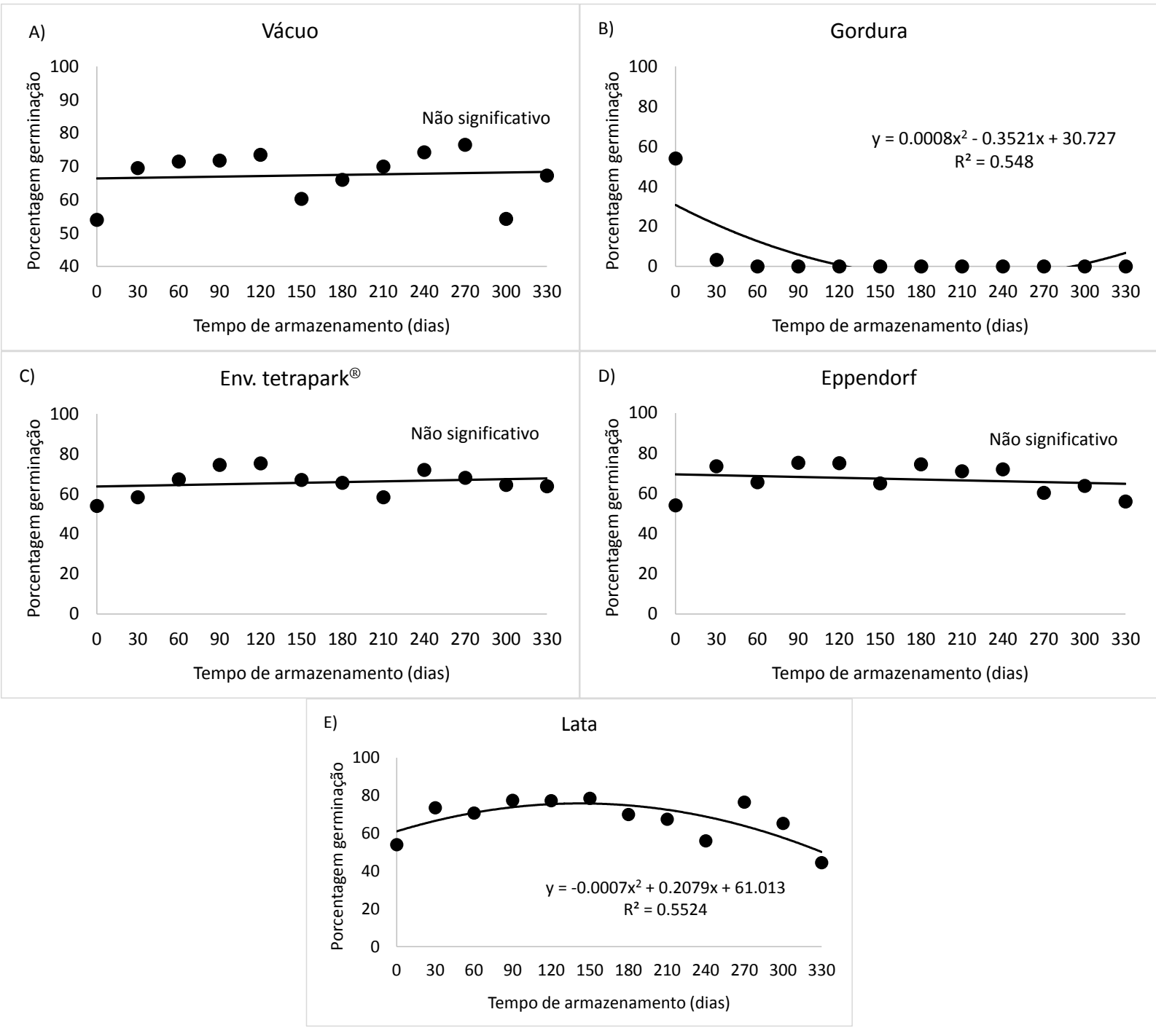

As embalagens de envelope Tetrapak, tubos eppendorf e lata de alumínio permitiram manter a viabilidade e consequentemente os níveis de germinação ao longo do armazenamento, com resultados mais estáveis, quando contrastados aos do armazenamento a vácuo ou em gordura animal. Tal resultado de manutenção da viabilidade pode ter relação com as condições favoráveis para a manutenção da semente com o menor volume de oxigênio, já que foram utilizadas embalagens com alta impermeabilidade dando origem à ambiente de atmosfera modificada, no qual aumenta o teor de $\mathrm{CO}_{2}$ e reduz o de $\mathrm{O}_{2}$ pelo processo respiratório (GONELI et al., 2005), permitindo a latência das sementes. Elevados teores de $\mathrm{O}_{2}$ durante $\mathrm{o}$ armazenamento de sementes de Euterpe edulis mantiveram o metabolismo acelerado ocasionando perda de vigor, contrário ao que ocorreu naqueles que propiciaram elevados teores de $\mathrm{CO}_{2}$ (RIBEIRO et al., 2010).

A perda de viabilidade quando armazenadas em gordura animal pode ter ocorrido pela total falta de oxigênio ao embrião ocasionado pelo isolamento da camada lipídica e hidrofóbica ao redor das sementes o que fez com que ocorresse o processo fermentativo, gerando a morte do mesmo.

Outro fator envolvido no armazenamento de sementes de araçazeiro é em relação ao seu teor de umidade. Silva et al. (2017) relataram que em excesso causa deterioração das reservas do embrião, reduzindo drasticamente a germinação, fato não ocorrido no presente trabalho, pois acredita-se que as embalagens testadas permitiram equilíbrio com ambiente externo reduzindo-se as perdas e consequentemente mantendo as sementes viáveis. 
Na embalagem a vácuo houve queda de germinação aos 150 e 180 dias. Podendo isto estar relacionado a espessura do saco de polietileno, ou até mesmo a ocorrência do rompimento da completa vedação, ocasionado certa troca gasosa, neste tempo específico pois estabeleceu superioridade nos demais períodos. Com isto percebe-se que esta técnica pode comprometer a viabilidade das sementes se não completamente vedada sua embalagem.

A conservação em lata de alumínio foi possível até os 300 dias de armazenamento, reduzindo-se a viabilidade após este período, mas esta redução não diferenciou-se estatisticamente quando contrastadas ao armazenamento em envelope Tetrapak, tubos Eppendorf ${ }^{\circ}$ ou em embalagens a vácuo.

O IVG apresentou comportamento semelhante a porcentagem de germinação, porém, houve maior variação entre os valores encontrados (Tabela 2).

Tabela 2. Índice de velocidade germinação (IVG) de sementes de Psidium catlleyanum armazenados em embalagem a vácuo, gordura animal, envelope Tetrapak', eppendorf e lata de alumínio por 330 dias com avaliações mensais.

\begin{tabular}{|c|c|c|c|c|c|}
\hline $\begin{array}{c}\text { Tempo } \\
\text { (dias) }\end{array}$ & Emb. Vácuo & Gordura & Env. Tetrapak ${ }^{\circledR}$ & Eppendorf & Lata alumínic \\
\hline 0 & $1,83 \mathrm{~d} A^{*}$ & 1,83 a $A$ & 1,83 e $A$ & $1,83 \mathrm{~b} \mathrm{~A}$ & 1,83 с A \\
\hline 30 & $3,09 a b c A$ & 1.07 b B & 2.72 bcde $A$ & 3.11 a $A$ & $3.23 \mathrm{ab} \mathrm{A}$ \\
\hline 60 & 3,72 a $A$ & 0,0 b B & 3.69 abc $A$ & 3.44 a A & 3.72 a A \\
\hline 90 & $3,50 a b A$ & 0,0 b B & $3.68 a b c A$ & 3.75 a A & 3.89 a A \\
\hline 120 & 3,63 a $A$ & 0,0 b B & 3.79 ab A & 3.40 a A & 3.97 a A \\
\hline 150 & $1,74 \mathrm{~dB}$ & $0,0 \mathrm{bC}$ & 2.30 de $A B$ & $2.73 \mathrm{ab} \mathrm{A}$ & $3.34 \mathrm{ab} \mathrm{A}$ \\
\hline 180 & $2,11 \mathrm{~cd} \mathrm{~B}$ & $0,0 \mathrm{bC}$ & 2,52 cde $A B$ & 3,18 а $A$ & $2,98 a b A B$ \\
\hline 210 & 3,87 a $A$ & 0,0 b B & 2,81 abcde $A$ & 3,77 a $A$ & $3,38 \mathrm{ab} \mathrm{A}$ \\
\hline 240 & 3,91 a A & 0,0 b B & 4,00 a $A$ & 3,68 a $A$ & 2,87 ab A \\
\hline 270 & 3,02 abc $A$ & $0,0 \mathrm{bC}$ & 2,56 cde $A B$ & 1,89 b B & 2,49 bc $A B$ \\
\hline 300 & $2,46 \mathrm{bcd} \mathrm{A}$ & $0,0 \quad b B$ & $3,33 \mathrm{abcd} A$ & $2,57 \mathrm{ab} A$ & 2,49 bc A \\
\hline 330 & $2,31 \mathrm{~cd} A$ & 0,0 b B & 2,24 de $A$ & $1,95 \mathrm{~b} \mathrm{~A}$ & $1,54 \subset A$ \\
\hline$V(\%)$ & & & 10,20 & & \\
\hline
\end{tabular}

*Médias seguidas por letras minúsculas distintas na coluna e maiúsculas na linha, diferem significativamente entre si pelo teste de Duncan $(\alpha=0,05)$.

As embalagens nos tubos da Eppendorf ${ }^{\circ} \mathrm{e}$ lata de alumínio apresentaram superioridade e regularidade dos dados de IVG quando comparadas as demais embalagens, com valores elevados até os 240 dias.

O envelope confeccionado com caixa Tetrapak ${ }^{\circ}$ apresentou superioridade para IVG aos $60,90,120,210,240,300$ dias. Comportamento semelhante foi observado na embalagem a vácuo, onde foi superior aos 30,60, 90, 120, 210, 240, 270 dias.

Todavia, comparando-se as embalagens, com exceção do uso de gordura animal, todas as demais não diferiram entre si para o IVG até os
120 dias e depois nos 210, 240, 300 e 330 dias. Nos 150 e 180 a superioridade foi obtido com envelope Tetrapak, Eppendorf ${ }^{\circ}$, e lata de alumínio e, aos 270 dias com vácuo, envelope Tetrapak, lata de alumínio.

O IVG das variáveis vácuo, envelope Tetrapak', tubo Eppendorf ${ }^{\circ}$ e lata de alumínio apresentaram significativas isoladamente com respostas quadráticas. A embalagem a vácuo alcançou o ponto de máxima em 147 dias, IVG de 3,48 , o envelope Tetrapak em 182 dias, IVG de 3,55 e quando conservados na lata de alumínio o ponto de máxima em 135 dias e IVG de 3,63. 
Figura 2. Índice de velocidade de germinação (IVG) em função do tempo de armazenamento em dias em vácuo (a), gordura (b), env. Tetrapak (c), eppendorf ${ }^{\circledR}$ (d), lata (e).
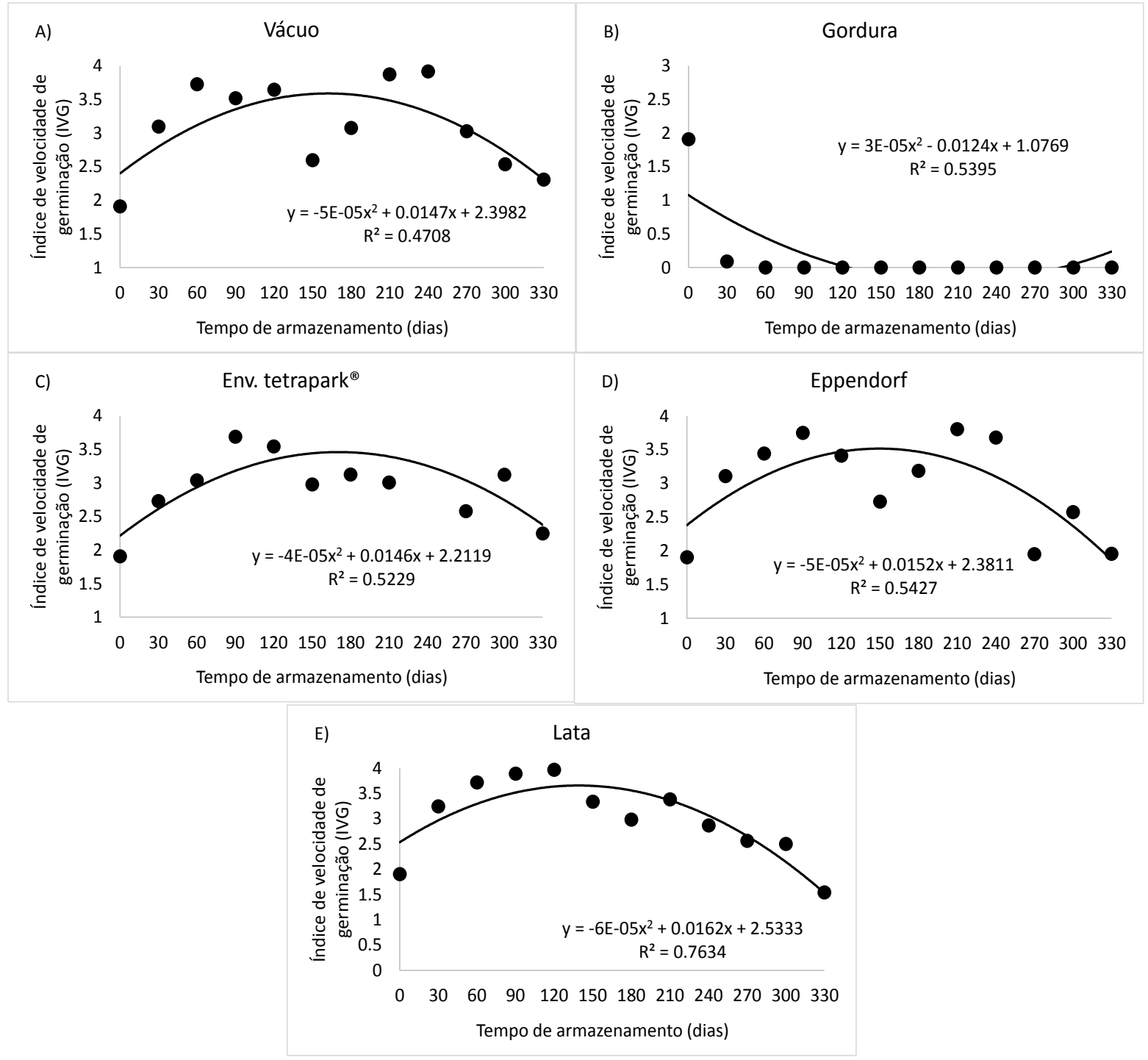

Para avaliação do TMG são preferíveis valores menores, o que demonstra maior uniformidade na germinação. As embalagens utilizadas para o armazenamento das sementes de araçazeiro apresentaram diferentes comportamentos para esta variável, destacandose o eppendorf ${ }^{\circ}$ e lata de alumínio, que mantiveram superioridade em relação as demais embalagens desde a primeira avaliação até os 240 dias (Tabela 3). 
Tabela 3. Tempo médio de germinação (TMG) de sementes de Psidium catlleyanum armazenados em embalagem a vácuo, gordura animal, envelope Tetrapak $^{\circledR}$, eppendorf e lata de alumínio por 330 dias com avaliações mensais.

\begin{tabular}{|c|c|c|c|c|c|}
\hline $\begin{array}{c}\text { Tempo } \\
\text { (dias) }\end{array}$ & Emb. Vácuo & Gordura & Env. Tetrapak ${ }^{\circledast}$ & Eppendorf & Lata alumínio \\
\hline 0 & 31,02 cde $A^{*}$ & 31,02 a $A$ & 31,02 de $A$ & $31,02 \mathrm{~cd} \mathrm{~A}$ & 31,02 bc $A$ \\
\hline 30 & 23,74 abc $A$ & $42,00 \quad b$ B & 18,59 ab A & 24,54 abc $A$ & 23,81 ab A \\
\hline 60 & 19,76 а $A$ & 0,0 с B & 22,50 abcd $A$ & 19,42 a $A$ & 19,65 a $A$ \\
\hline 90 & 21,13 ab A & 0,0 с B & 21,01 abc $A$ & 20,46 ab A & 20,52 a $A$ \\
\hline 120 & 21,18 ab A & $0,0 \mathrm{cB}$ & 20,95 abc A & 22,80 abc $A$ & 20,39 a A \\
\hline 150 & 37,61 e $B$ & $0,0 \subset \mathrm{C}$ & 32,75 e $A B$ & $24,43 a b c A$ & $24,38 \mathrm{ab} \mathrm{A}$ \\
\hline 180 & 34,37 de $B$ & $0,0 \subset C$ & 27,24 cde $A B$ & $23,44 a b c A$ & 23,66 ab A \\
\hline 210 & 18,44 a $A$ & 0,0 с B & 20,45 abc $A$ & 19,36 а $A$ & 20,64 a A \\
\hline 240 & 19,59 а $A$ & $0,0 \subset B$ & 18,16 a $A$ & 20,08 ab A & 19,84 a $A$ \\
\hline 270 & 25,42 abcd $A$ & 0,0 cD & 26,96 bcde $A B$ & $36,35 \mathrm{dC}$ & 34,84 c BC \\
\hline 300 & $22,49 a b c A B$ & $0,0 \subset C$ & 21,46 abc $A$ & 28,40 bcd $A B$ & 29,99 bc $B$ \\
\hline 330 & 29,32 bcde $A$ & $N G \quad c B$ & 28,60 cde A & 28,76 bcd A & 29,29 bc A \\
\hline
\end{tabular}

* Médias seguidas por letras minúsculas distintas na coluna e maiúsculas na linha, diferem significativamente entre si pelo teste de Duncan $(\alpha=0,05)$.

Figura 3. Tempo médio de germinação (TMG) em função do tempo de armazenamento em dias em vácuo (a), gordura (b), env. Tetrapak $^{\circledast}$ (c), eppendorf ${ }^{\circledast}$ (d), lata (e).
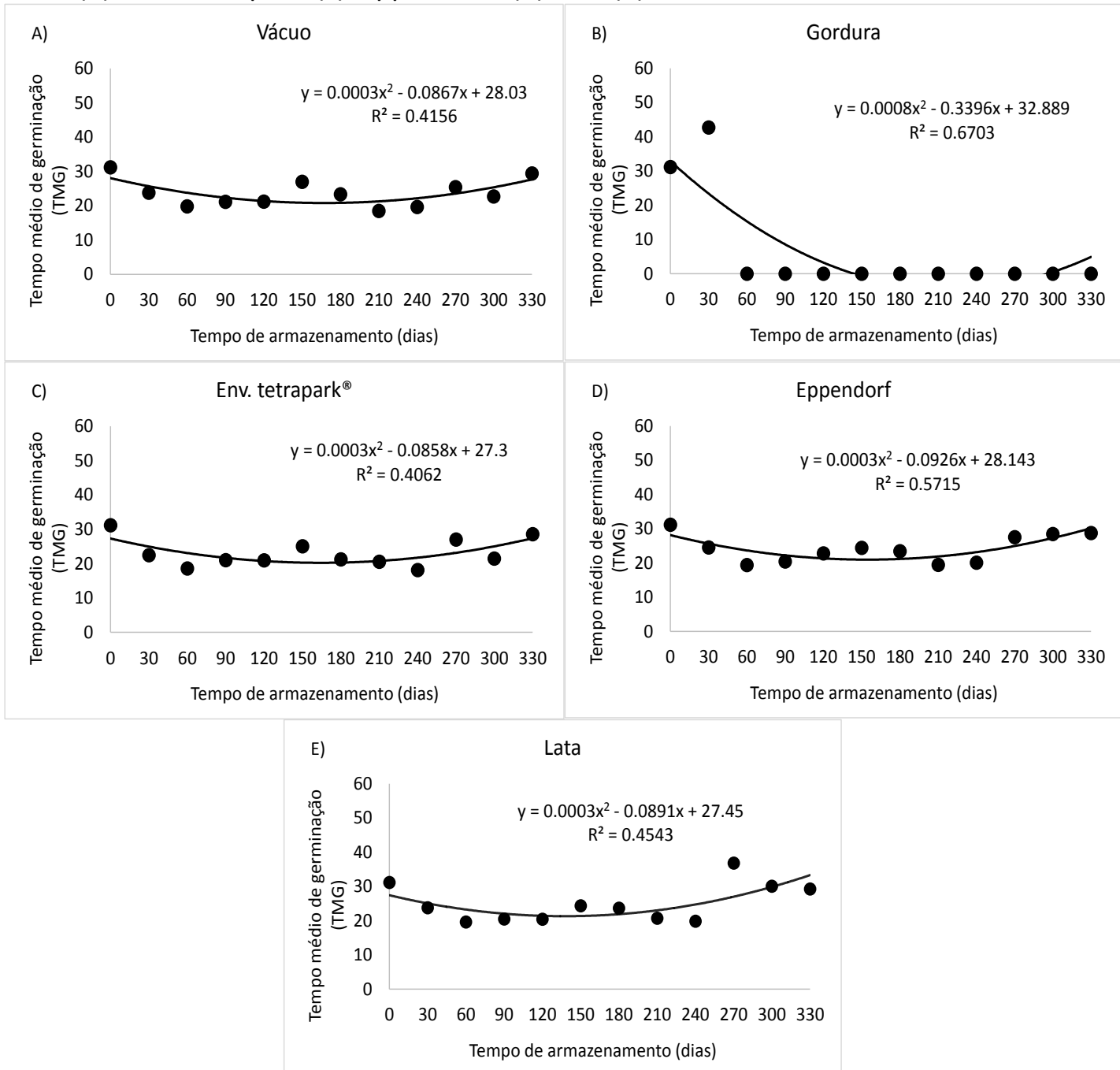
Os TMG quando observados de maneira isolada apresentou efeitos quadráticos significativos estatisticamente. Os valores de 144 dias, TMG de 22, 143 dias, TMG de 21, 154 dias, TMG de 21 e 149 dias TMG de 21 foram para vácuo, envelope Tetrapak ${ }^{\circ}$, tubo eppendorf ${ }^{\circ}$ e lata de alumínio, respectivamente.

O armazenamento de semente de araçazeiro em gordura não se mostrou viável já que os valores obtidos de germinação, IVE ou TMG foram insatisfatórios. A embalagem a vácuo apresentou resultados promissores, porém, em alguns períodos inconsistentes o que requer melhorias neste tipo de embalagem.

Os resultados deste trabalho foram superiores aos encontrados por Silva et al. (2017), que também trabalharam com sementes de araçazeiro $\mathrm{Ya}-\mathrm{C} y$ e obtiveram resultados superiores de germinação (maior que $50 \%$ ) com apenas 30 dias de armazenamento e ausência de germinação após os 90 dias. Este fato pode ter ocorrido pela não utilização de embalagens apropriadas para 0 armazenamento das sementes, demonstrando que a embalagem correta pode propiciar maior longevidade das sementes desta espécie.

\section{CONCLUSÃO}

É recomendado o uso de recipientes como eppendorf ${ }^{\circledast}$ ou material Tetrapak $^{\circledR}$ para armazenar sementes de araçazeiro $\mathrm{Ya}-\mathrm{Cy}$, visando conservar sua viabilidade e vigor até os 330 dias.

Utilização de gordura animal não foi recomendada para armazenamento de sementes de araçazeiro Ya-Cy.

\section{REFERÊNCIAS}

BRACK, P.; KINUPP, V. F.; SOBRAL, M. E. G. Levantamento preliminar de espécies frutíferas de árvores e arbustos nativos com uso atual ou potencial do Rio Grande do Sul. Revista brasileira de Agroecologia, v. 2, n. 1, p. 1769-1772, 2007.

BRASIL. Regras para análise de sementes. Ministério da Agricultura, Pecuária e Abastecimento. Secretaria de Defesa Agropecuária. Brasília: Mapa/ACS, 2009. 398 p.

BOX, G.; COX, D. An analysis of transform. Journal of the Royal Statistical Society, v. 26, n.2, p. 211252, 1964. http://www.jstor.org/stable/2984418
CASTRO, R.D.; BRADFORD, K.J.; HILHORST, H.W.M. Desenvolvimento de sementes e conteúdo de água. In: FERREIRA, A.G.; BORGHETTI, F. (Org.). Germinação: do básico ao aplicado. Porto Alegre: Artmed, 2004. p.51-67.

CISNEIROS, R. A.; MATOS, V. P.; LEMOS, M. A.; REIS, O. V.; QUEIROZ, R. D. M. Qualidade fisiológica de sementes de araçazeiro durante o armazenamento. Revista Brasileira de Engenharia Agrícola e Ambiental, v. 7, n. 3, p. 513-518, 2003. http://dx.doi.org/10.1590/S141543662003000300018.

DANNER, M. A. Diagnóstico ecogeográfico e caracterização morfogenética de jabuticabeiras. 2009. 130 f Dissertação (Mestrado) Universidade Tecnológica Federal do Paraná, Pato Branco-PR, 2009.

DANNER, M. A.; CITADIN, I.; SASSO, S. A. Z.; SACHET, M. R.; AMBRÓSIO, R. Fenologia da floração e frutificação de mirtáceas nativas da floresta com araucária. Comunicação científica. Revista Brasileira de Fruticultura, Jaboticabal, v. 32, n. 1, p. 291-295, 2010. https://doi.org/10.1590/S0100$\underline{29452010005000008}$

FRANZON, R. C. Caracterização de mirtáceas nativas do sul do Brasil. 2004. 114f. Dissertação (Mestrado) - Curso de Pós-Graduação em Agronomia, Faculdade de Agronomia Eliseu Maciel, Universidade Federal de Pelotas, PelotasRS, 2004.

FRANZON, R. C.; CAMPOS, L. Z. O.; PROENÇA, C. E. B.; SOUZA-SILVA, J. C. Araçás do Gênero Psidium: Principais espécies, ocorrência, descrição e usos. Planaltina: Embrapa Cerrados, 2009. (Documento, 266).

GONELI, A. L. D.; CORRÊA, P. C.; DIAS, D. C. F. S.; MIRANDA, G. V. Efeito da danificação mecânica na qualidade fisiológica de sementes de milhopipoca durante o armazenamento. Revista Brasileira de Produtos Agroindustriais, Campina Grande, v. 7, n. 2, p. 101-111, 2005. https://doi.org/10.15871/15178595/rbpa.v7n2p101-111

KOHAMA, S.; MALUF, A. M.; BILIA, D. A. C.; BARBEDO, C. J. Secagem e armazenamento de 
sementes de Eugenia brasiliensis LAM. (Grumixameira). Revista Brasileira de Sementes, v. $28, \quad$ n. $1, \quad$ p. $72-78,2006$. https://doi.org/10.1590/S0101-

\section{0}

MAGUIRE, J. D. Speed of germination: aid in selection and evaluation for seedling emergence and vigor. Crop Science, Madison, v. 2, n. 1, p. 176-177, 1962. https://doi.org/10.2135/cropsci1962.0011183X0 $00200020033 x$

MANICA, I. Frutas nativas, silvestres e exóticas 1. Técnicas de produção e mercado: abiu, amorapreta, araçá, bacuri, biribá, carambola, cereja-doriogrande, jaboticaba. Porto Alegre: Cinco Continentes, $2000.2327 p$. https://doi.org/10.1590/S1415$\underline{43662003000300018}$

NERI-NUMA, I. A.; CARVALHO-SILVA, L. B.; MORALES, J. P.; MALTA, L. G.; MURAMOTO, M. T.; FERREIRA, J. E.; CARVALHO, J. E.; RUIZ, A. L.; MARÓSTICA JUNIOR, M. R.; PASTORE, G. M. Evaluation of the antioxidant, antiproliferative and antimutagenic potential of araçá-boi fruit (Eugenia stipitata Mc Vaugh - Myrtaceae) of the Brazilian Amazon Forest. Food Research International, v. 50, 1. ed., p. 70-76, 2013. https://doi.org/10.1016/j.foodres.2012.09.032

VIEIRA, R. F.; AGOSTINI-COSTA, T. S.; SILVA, D. B.; SANO, S. M.; FERREIRA, F. R. Frutas nativas da região centro-oeste. Embrapa Recursos Genéticos e Biotecnologia, 2006.

WIELEWICKI, A.P.; LEONHARDT, C.; SCHLINDWEIN, G.; MEDEIROS, A.C.S. Proposta de padrões de germinação e teor de água para sementes de algumas espécies florestais presentes na região sul do Brasil. Revista Brasileira de Sementes, v.28, p.191-197, 2006. http://dx.doi.org/10.1590/S010131222006000300027.
$R$ CORE TEAM. R: A language and environment for statistical computing. 2017. R Foundation for Statistical Computing, Vienna, Austria. Disponível em: <https://www.R-project.org/>.

RIBEIRO, M. S.; STEFFENS, C. A.; AMARANTE, C. V. T.; PIKART, T. G.; OLIVEIRA, L. M. Armazenamento de sementes de palmiteiro sob atmosfera modificada. Bragantia, Campinas, v. 69, n. 4, p.989-994, 2010. https://doi.org/10.1590/S0006$\underline{87052010000400026}$

SILVA, M.; RADAELLI, J. C.; MOURA, G. C.; KOSERA NETO, C.; STEFENI, A. R.; PORTO, A. H.; FOQUESATTO, C. F.; WAGNER JÚNIOR, A. Hydrocondicionament and storage of yellow araçazeiro seeds. Australian Journal of Basic and Applied Sciences, v. 11, n. 2, p. 115-122, 2017.

TOMAZ, Z. F. P.; GALARÇA, S. P.; LIMA, C. S. M. Tratamentos pré-germinativos em sementes de araçazeiro (Psidium cattleyanum Sabine L.). Revista Brasileira de Agrociência, v. 17, n 1-4, p. 60-65, 2011.

ZANELA, J.; WAGNER JÚNIOR, A.; CASSOL, D. A.; ALEGRETTI, A. L.; PIROLA, K.; MAZARO, S. M. Biofilme e pré-embebição de sementes na germinação do araçazeiro 'Ya-Cy'. Revista Brasileira de Agrociência, v. 18, n. 2-4, p. 229-232, 2012.

Recebido para publicação em 26/09/2017

Revisado em 24/03/2018

Aceito em 28/03/2018 